\title{
Metastable gravity on classical defects
}

\author{
Christophe Ringeva** \\ Blackett Laboratory, Imperial College, Prince Consort Road, London SW7 2AZ, United Kingdom \\ Jan-Willem Rombouts $\dagger$ \\ Department of Physics, New York University, 4 Washington Place, New York, NY 10003, USA
}

(Dated: December 6, 2018)

\begin{abstract}
We discuss the realization of metastable gravity on classical defects in infinite-volume extra dimensions. In dilatonic Einstein gravity, it is found that the existence of metastable gravity on the defect core requires violation of the Dominant Energy Condition for codimension $N_{\mathrm{c}}=2$ defects. This is illustrated with a detailed analysis of a six-dimensional hyperstring minimally coupled to dilaton gravity. We present the general conditions under which a codimension $N_{\mathrm{c}}>2$ defect admits metastable modes, and find that they differ from lower codimensional models in that, under certain conditions, they do not require violation of energy conditions to support quasi-localized gravity.
\end{abstract}

PACS numbers: 04.50.+h, 11.10.Kk, 98.80.Cq

\section{INTRODUCTION}

In recent years, there has been great interest in models of infinite-volume extra dimensions. The Dvali-Gabadadze-Porrati (DGP) model realizes fourdimensional $(4 D)$ gravity on a membrane in a flat five-dimensional $(5 D)$ bulk by adding a large, induced Einstein-Hilbert term to the worldvolume of a threebrane [1]. The Einstein-Hilbert term on the brane is generically induced by quantum loops of matter fields on the brane, and realizations of this mechanism are known in more fundamental theories like string theory [2, 3, 4]. One of the main phenomenological attractions of DGP is its celebrated "self-accelerated" cosmological solution, giving small late-time acceleration without the need to invoke dark energy [5]. Higher codimensional realizations of DGP gravity are known to be non-trivial, because a straightforward generalization of the model gives singular propagation and other difficulties $6,7,8,9,10,11$.

On the other hand, codimension $N_{\mathrm{c}}>1$ theories have very attractive properties, foremost because of their unique way of addressing the cosmological constant problem [8, 9]. Being non-local in the infrared, these models evade Weinberg's no-go theorem, unlike compact extra dimensional theories. The idea put forward in [8] for example is that, in infinite-volume extra dimensional theories, one can have $4 D$ nearly flat solutions even though the brane tension is large ("the $4 D$ cosmological constant curves the bulk, not the brane"). In more detail, using the induced gravity setting, it was argued that there are solutions of supercritical branes in infinite bulk with tension $\mathcal{T}>M_{*}^{4}$ that support a regular geometry if one

\footnotetext{
*Electronic address: c.ringeval@imperial.ac.uk
}

†Electronic address: jwr218@nyu.edu allows for an inflating worldvolume with Hubble rate:

$$
H \sim M_{*}\left(\frac{M_{*}^{4}}{\mathcal{T}}\right)^{1 /\left(N_{\mathrm{c}}-2\right)}
$$

$M_{*}$ is the fundamental higher dimensional Planck mass (constrained to be quite low $\sim 10^{-3} \mathrm{eV}$ ) and $N_{\mathrm{c}}$ the number of codimensions. In predicting an inflation rate inversely proportional to the vacuum energy density these models provide an interesting way of solving the cosmological constant problem.

In Refs. [10, 11, 12, 13] different ways of realizing DGP-like gravity in higher dimensional context were discussed. A fundamental difference with the codimension one model is that $4 D$ gravity is mediated there by massive resonant graviton states, with the width of the resonance much smaller than its mass 11] (DGP allows $4 D$ gravity by a broad resonance peaked around zero mass). However, the general task remains to find a physical framework where gravitational dynamics can be approximated by a DGP-like action

$$
S=M_{\mathrm{p}}^{2} \int \sqrt{|\bar{g}|} \bar{R} \mathrm{~d}^{4} x+M_{*}^{2+N_{\mathrm{c}}} \int \sqrt{|g|} R \mathrm{~d}^{N_{\mathrm{c}}+4} X .
$$

Here $\bar{g}$ and $\bar{R}$ are the determinant and scalar curvature of the induced metric on the brane while $g$ and $R$ are the same quantities for the bulk metric.

In analogy with the action (2), in Ref. 11], 4D-like gravity is realized by a "regularized" action:

$$
S=M_{*}^{2+N_{\mathrm{c}}} \int \sqrt{|g|} R \mathcal{F}(X) \mathrm{d}^{N_{\mathrm{c}}+4} X
$$

where $\mathcal{F}$ is a sharply peaked profile around the origin of the transverse space, which can be interpreted as a varying Planck mass in the transverse dimensions. This action has the same features as Eq. (2) in that gravity in the brane core is weakly coupled while the bulk is strongly coupled. It was shown that by appropriately choosing the profile $\mathcal{F}$, one propagates $4 D$ gravity by 
resonances in the brane core ${ }^{1}$. Indeed, the Newtonian potential between localized sources on the brane, at a dimensionless distance $x$ from eachother, can be obtained by considering the exchange of Kaluza-Klein modes 14 , 15, 16

$$
V_{\mathrm{g}}(x) \propto \int_{0}^{\infty} \rho(\bar{M}) \frac{\mathrm{e}^{-\bar{M} x}}{x} \mathrm{~d} \bar{M} .
$$

Here $\rho(\bar{M})$ is the spectral density on the brane, which is modified from its empty space value in the presence of quasi-bound states. For instance, in flat $5 D$ spacetime, one recovers a static potential varying as $1 / x^{2}$ from a constant spectral density. One can consider the case in which the spectral density is, in addition to its standard behavior, strongly peaked around a particular mass $\bar{M}_{\mathrm{c}}$

$$
\rho(\bar{M})=1+\delta\left(\bar{M}-\bar{M}_{\mathrm{c}}\right) .
$$

From Eq. (4), this would lead to a gravitational potential

$$
V_{\mathrm{g}}(x) \propto \frac{1}{x}\left(\mathrm{e}^{-\bar{M}_{\mathrm{c}} x}+\frac{1}{x}\right),
$$

and one recovers $4 D$-like gravity on the scales $1 \ll x \ll$ $1 / \bar{M}_{\mathrm{c}}$, which is physically attractive for $\bar{M}_{\mathrm{c}} \ll 1$. Interestingly, such systems predict generically an infrared as well as ultraviolet modification of gravity. In the regularization of 11] this behaviour was indeed recovered, using appropriate regulating profiles for the action Eq. (31).

From Eq. (2), it is clear that the previous mechanism could also be realized by letting $\sqrt{|g|}$ vary transverse to the brane instead of the Planck mass. This has been studied in Refs. 17, 18] in the context of warped extra dimensions, with or without asymptotic flatness.

Following the previous discussing, we want to focus in this paper on the following question [19, 20]: is it possible to realize $4 D$ gravity by (meta)stable states in the context of classical field theories? Topological defects serve as natural branes in non-perturbative field theory [21, 22, 23, 24, 25] and they have been intensively studied in the context of warped extra dimensions 26, 27, 28, 29], while an obvious setting to generate a varying Planck mass is dilatonic gravity 30, 31, 32, 33]. The formulation of these theories using an underlying non-linear sigma model realization is important to address their (supersymmetric) phenomenology [19] and internal consistency (for debate on the DGP model see e.g. Refs. 34, 35, 36, 37]).

Indeed, the action (3) suggests that in an appropriately formulated theory of dilatonic gravity, one might achieve metastable states in the core of a topological defect if the dilaton condenses strongly around the core and

\footnotetext{
1 The varying gravitational coupling constant defined by $\mathcal{F}$ is analogous to a varying dielectric constant in electrostatics. The appearance of resonant states is supported by this analogy.
}

falls off sharply outside the defect. Clearly, such a model would involve both variations in the metric through the defect energy-momentum tensor, and variations of the "bulk Planck mass" by means of the dilaton. It is not clear a priori how these effects are balanced, and one has to construct explicit solutions to make quantitative statements about their properties.

This paper is organized as follows. We first construct an illustrative example of a local hyperstring in six dimensional dilatonic gravity. Considering a massive dilaton, we find that the dilaton naturally condenses around the hyperstring core, in a region set by its Compton wavelength, and that the geometry of the system is asymptotically flat. The full non-linear solution is computed numerically. Next we study the propagation of $4 D$ tensor perturbations on this background and find that there are no metastable states appearing in this setting.

We then ask under which general conditions (on the defect forming matter and gravity content) tensor (with respect to the $4 D$ brane) resonant states exist in the defect core in infinite-volume extra dimensions. It will appear that a violation of the Dominant Energy Condition (DEC) is a necessary condition for this to happen in codimension $N_{\mathrm{c}}=2$.

In the last section we restate the same question for the case of codimension $N_{\mathrm{c}}>2$ defects. It is shown that, at least under reasonable conditions, metastable gravity is allowed without violation of positive energy conditions. We leave however explicit constructions for future work.

Finally we conclude, and stress that our results only concern classical field theoretical realizations of metastable gravity. As mentioned before, there exist a number of different ways towards realizing metastable gravity on branes in infinite-volume extra dimensions, using the DGP loop induced effects or explicit constructions in string theory. Our work evidently does not address those models.

\section{4D HYPERSTRING COUPLED TO DILATONIC GRAVITY}

In the following we consider the action for a fourdimensional hyperstring coupled to scalar-tensor gravity in a six-dimensional spacetime [30, 31]:

$$
\begin{aligned}
S & =\int \frac{1}{2 \kappa_{6}^{2}} \mathrm{e}^{\phi}\left[R-g^{A B} \partial_{A} \phi \partial_{B} \phi-U(\phi)\right] \sqrt{|g|} \mathrm{d}^{6} x \\
& +\int \mathcal{L}_{\text {mat }} \sqrt{|g|} \mathrm{d}^{6} x,
\end{aligned}
$$

where $g_{A B}$ is the $6 D$ metric with signature $(-,+,+, \ldots)$, $R$ its Ricci scalar, $\phi$ the dilaton field with a potential $U(\phi), \kappa_{6}^{2} \equiv 32 \pi^{2} G_{6} / 3, G_{6}$ being the 6-dimensional gravity constant. In order to allow for topological vortex configurations, we include a complex scalar field $\Phi$ :

$$
\mathcal{L}_{\text {mat }}=-\frac{1}{2} g^{A B}\left(\mathcal{D}_{A} \Phi\right)^{\dagger} \mathcal{D}_{B} \Phi-V(\Phi)-\frac{1}{4} H_{A B} H^{A B},
$$


in which capital Latin indexes $A, B \ldots$ run from 0 to 5 and $H_{A B}$ is the electromagnetic-like tensor defined by

$$
H_{A B}=\partial_{A} C_{B}-\partial_{B} C_{A},
$$

where $C_{B}$ is the 1 -form connection. The $\mathrm{U}(1)$ covariant derivative $\mathcal{D}_{A}$ is defined by

$$
\mathcal{D}_{A} \equiv \partial_{A}-i q C_{A},
$$

where $q$ is the charge. The potential of the scalar field $\Phi$ is chosen to break the underlying $U(1)$ symmetry and thereby allow for hyperstring configurations,

$$
V(\Phi)=\frac{\lambda}{8}\left(|\Phi|^{2}-\eta^{2}\right)^{2},
$$

where $\lambda$ is a coupling constant and $\eta=\langle|\Phi|\rangle$ is the magnitude of the scalar field vacuum expectation values (VEV).

The variations of the action (77) with respect to the metric and the dilaton field lead to the equations of motion in the scalar-tensor gravity sector, in the Jordan frame one gets

$$
\begin{aligned}
G_{A B} & =\mathrm{e}^{-\phi} \kappa_{6}^{2} T_{A B}+2 \partial_{A} \phi \partial_{B} \phi-\frac{1}{2} g_{A B}\left[3 \partial_{X} \phi \partial^{X} \phi\right. \\
& +U(\phi)]+\nabla_{A} \partial_{B} \phi-g_{A B} \nabla_{X} \partial^{X} \phi \\
\nabla_{X} \partial^{X} \phi & =\frac{1}{2} U(\phi)+\frac{1}{2} \frac{\mathrm{d} U}{\mathrm{~d} \phi}-\frac{1}{2} R-\frac{1}{2} \partial_{X} \phi \partial^{X} \phi
\end{aligned}
$$

where $G_{A B}$ is the $N$-dimensional Einstein tensor and $T_{A B}$ the stress tensor of the hyperstring:

$$
T_{A B} \equiv-2 \frac{\delta \mathcal{L}_{\text {mat }}}{\delta g^{A B}}+g_{A B} \mathcal{L}_{\text {mat }} .
$$

The metric is chosen so as to respect the cylindrical static symmetry in the two extra dimensions and Poincaré invariance along the remaining four spacetime coordinates;

$$
\mathrm{d} s^{2}=g_{A B} \mathrm{~d} x^{A} \mathrm{~d} x^{B}=\mathrm{e}^{\sigma(r)} \eta_{\mu \nu} \mathrm{d} x^{\mu} \mathrm{d} x^{\nu}+\mathrm{d} r^{2}+\omega^{2}(r) \mathrm{d} \theta^{2},
$$

where $\eta_{\mu \nu}$ is the four dimensional Minkowski metric of signature $(-,+,+,+)$, and $(r, \theta)$ the polar coordinates in the extra dimensions. Greek indexes $\mu, \nu \ldots$ run from 0 to 3 and describe the brane worldvolume.

We chose the Nielsen-Olesen ansatz for the matter field leading to an hyperstring configuration with unit winding number [29, 38],

$$
\Phi=\varphi(r) \mathrm{e}^{i \theta}, \quad C_{\theta}=\frac{1}{q}[1-Q(r)] .
$$

The only non-vanishing component of the electromagnetic tensor is $H_{\theta r}=Q^{\prime} / q$. The dilaton is also assumed to respect the cylindrical symmetry and depends only on the extra radial coordinates $\phi(r)$.

\section{A. Background Solution}

In terms of the dimensionless coordinate

$$
\rho \equiv m_{\mathrm{h}} r=\sqrt{\lambda} \eta r,
$$

$m_{\mathrm{h}}$ being the mass of the Higgs boson, the Einstein tensor reads

$$
\begin{aligned}
G_{\mu \nu} & =g_{\mu \nu} m_{\mathrm{h}}^{2}\left(\frac{3}{2} \ddot{\sigma}+\frac{3}{2} \dot{\sigma}^{2}+\frac{\ddot{\omega}}{\omega}+\frac{3}{2} \dot{\sigma} \frac{\dot{\omega}}{\omega}\right) \\
G_{r r} & =m_{\mathrm{h}}^{2}\left(\frac{3}{2} \dot{\sigma}^{2}+2 \dot{\sigma} \frac{\dot{\omega}}{\omega}\right) \\
G_{\theta \theta} & =\omega^{2} m_{\mathrm{h}}^{2}\left(2 \ddot{\sigma}+\frac{5}{2} \dot{\sigma}^{2}\right)
\end{aligned}
$$

where the dot stands for differentiation with respect to $\rho$. From Eqs. (8) and (14), the stress tensor reads

$$
\begin{aligned}
& T_{\mu \nu}=-g_{\mu \nu}\left(\frac{1}{2} m_{\mathrm{h}}^{2} \dot{\varphi}^{2}+\frac{1}{2} \frac{\varphi^{2} Q^{2}}{\omega^{2}}+V(\varphi)+\frac{1}{2} \frac{m_{\mathrm{h}}^{2} \dot{Q}^{2}}{q^{2} \omega^{2}}\right), \\
& T_{r r}=\frac{1}{2} m_{\mathrm{h}}^{2} \dot{\varphi}^{2}-\frac{1}{2} \frac{\varphi^{2} Q^{2}}{\omega^{2}}-V(\varphi)+\frac{1}{2} \frac{m_{\mathrm{h}}^{2} \dot{Q}^{2}}{q^{2} \omega^{2}}, \\
& T_{\theta \theta}=\omega^{2}\left[-\frac{1}{2} m_{\mathrm{h}}^{2} \dot{\varphi}^{2}+\frac{1}{2} \frac{\varphi^{2} Q^{2}}{\omega^{2}}-V(\varphi)+\frac{1}{2} \frac{m_{\mathrm{h}}^{2} \dot{Q}^{2}}{q^{2} \omega^{2}}\right] .
\end{aligned}
$$

In terms of dimensionless quantities, the Einstein and dilaton equations of motion (12) and (13) can be recast into

$$
\begin{aligned}
& \frac{3}{2} \ddot{\sigma}+\frac{3}{2} \dot{\sigma}^{2}+\frac{\ddot{\varpi}}{\varpi}+\frac{3}{2} \dot{\sigma} \frac{\dot{\varpi}}{\varpi}=\alpha \mathrm{e}^{-\phi}\left[-\dot{f}^{2}-\frac{f^{2} Q^{2}}{\varpi^{2}}\right. \\
& \left.-\frac{1}{4}\left(f^{2}-1\right)^{2}-\frac{\dot{Q}^{2}}{\varepsilon \varpi^{2}}\right]-\ddot{\phi}-\frac{3}{2} \dot{\phi}^{2} \\
& -\left(\frac{3}{2} \dot{\sigma}+\frac{\dot{\varpi}}{\varpi}\right) \dot{\phi}-\frac{1}{2} \frac{U}{m_{\mathrm{h}}^{2}} \\
& \frac{3}{2} \dot{\sigma}^{2}+2 \dot{\sigma} \frac{\dot{\varpi}}{\varpi}=\alpha \mathrm{e}^{-\phi}\left[\dot{f}^{2}-\frac{f^{2} Q^{2}}{\varpi^{2}}-\frac{1}{4}\left(f^{2}-1\right)^{2}+\frac{\dot{Q}^{2}}{\varepsilon \varpi^{2}}\right] \\
& +\frac{1}{2} \dot{\phi}^{2}-\left(2 \dot{\sigma}+\frac{\dot{\varpi}}{\varpi}\right) \dot{\phi}-\frac{1}{2} \frac{U}{m_{\mathrm{h}}^{2}}, \\
& 2 \ddot{\sigma}+\frac{5}{2} \dot{\sigma}^{2}=\alpha \mathrm{e}^{-\phi}\left[-\dot{f}^{2}+\frac{f^{2} Q^{2}}{\varpi^{2}}-\frac{1}{4}\left(f^{2}-1\right)^{2}+\frac{\dot{Q}^{2}}{\varepsilon \varpi^{2}}\right] \\
& -\ddot{\phi}-\frac{3}{2} \dot{\phi}^{2}-2 \dot{\sigma} \dot{\phi}-\frac{1}{2} \frac{U}{m_{\mathrm{h}}^{2}} \\
& \ddot{\phi}+\frac{1}{2} \dot{\phi}^{2}+\left(2 \dot{\sigma}+\frac{\dot{\varpi}}{\varpi}\right) \dot{\phi}=\frac{1}{2 m_{\mathrm{h}}^{2}}\left[U+\frac{\mathrm{d} U}{\mathrm{~d} \phi}-R\right] .
\end{aligned}
$$


We have defined the dimensionless parameters

$$
\alpha \equiv \frac{1}{2} \kappa_{6}^{2} \eta^{2}, \quad \beta \equiv \frac{m_{\mathrm{d}}^{2}}{m_{\mathrm{h}}^{2}}, \quad \varepsilon \equiv \frac{m_{\mathrm{b}}^{2}}{m_{\mathrm{h}}^{2}}=\frac{q^{2} \eta^{2}}{\lambda \eta^{2}}
$$

where $m_{\mathrm{b}}$ is the mass of the gauge vector boson and $m_{\mathrm{d}}$ the mass of the dilaton in the Einstein frame for

$$
U(\phi)=m_{\mathrm{d}}^{2} \phi^{2} \mathrm{e}^{\phi / 2} .
$$

The Ricci scalar is given by

$$
R=-m_{\mathrm{h}}^{2}\left(4 \ddot{\sigma}+5 \dot{\sigma}^{2}+2 \frac{\ddot{\varpi}}{\varpi}+4 \dot{\sigma} \frac{\dot{\varpi}}{\varpi}\right),
$$

and $\varpi$ is the dimensionless angular metric factor

$$
\varpi \equiv m_{\mathrm{h}} \omega
$$

The modulus of the Higgs field appears through the dimensionless function $f(\rho)$

$$
f \equiv \frac{\varphi}{\eta}
$$

Note that the metric (15) has no conical singularity in $r=0$ provided $\omega \sim r$, i.e. $\varpi \sim \rho$ in the hyperstring core. Differentiation of the action (17) with respect to the Higgs field yields the Klein-Gordon equation

$$
\ddot{f}+\left(2 \dot{\sigma}+\frac{\dot{\varpi}}{\varpi}\right) \dot{f}-\frac{f Q^{2}}{\varpi^{2}}-\frac{1}{2} f\left(f^{2}-1\right)=0,
$$

while differentiation with respect to the gauge field gives the Maxwell equation

$$
\ddot{Q}+\left(2 \dot{\sigma}-\frac{\dot{\varpi}}{\varpi}\right) \dot{Q}-\varepsilon f^{2} Q=0 .
$$

Since the dilaton is only coupled to the metric, the Bianchi identities ensure that stress-energy conservation is still verified in the matter sector. Therefore, there is a redundant equation in the system of differential equations (20) to (30) and we choose to solve numerically Eqs. (20), (22), (23), (29) and (30).

As usual, the Higgs field vanishes in the defect core, i.e. $\Phi=0$ for $\rho=0$, while it relaxes to its vacuum expectation value $(\mathrm{VEV}), \eta$, in the bulk. These conditions translate into the following boundary conditions for the function $f$ :

$$
f(0)=0, \quad \lim _{\rho \rightarrow+\infty} f=1 .
$$

The corresponding boundary conditions for the gauge field are given by

$$
Q(0)=1, \quad \lim _{\rho \rightarrow+\infty} Q=0 .
$$

Moreover, we assume that no additional $\delta$-like energy distribution lies in the hyperstring core. The geometry is

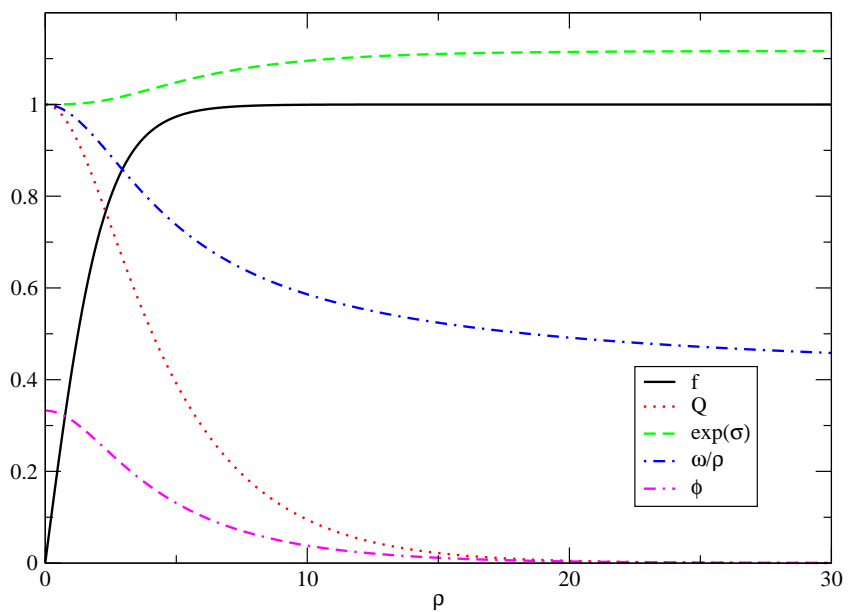

FIG. 1: Transverse profiles of the dimensionless hyperstring forming Higgs $f$ and gauge field $Q$, together with the metric factors $\mathrm{e}^{\sigma}$ and $\varpi / \rho$ for the parameters $\alpha=0.4, \beta=0.1$ and $\varepsilon=0.1$. The dilaton $\phi$ condenses in the hyperstring core and the spacetime has an asymptotically flat (conical) geometry.

therefore regular in the core and the metric coefficients verify

$$
\begin{aligned}
\sigma(0) & =0, \quad \dot{\sigma}(0)=0, \\
\varpi(0) & =0, \quad \dot{\varpi}(0)=1 .
\end{aligned}
$$

Asymptotically we are interested in the cosmic string branch solutions. Recall that the dilaton is massive and does not couple to the matter sector allowing the existence of asymptotic flat conical spacetimes without long range dilatonic effects $[39,40,41,42]$. This leads to the boundary conditions

$$
\dot{\phi}(0)=0, \quad \lim _{\rho \rightarrow+\infty} \phi=0,
$$

where the constraint at $\rho=0$ comes from the regularity requirement of a non-winding field. The numerical method used to solve this system is described in appendix $\mathrm{A}$ and a typical solution is presented in Fig. 11 The dilaton condenses in the hyperstring core while the spacetime geometry is asymptotically conical, with a missing angle given by the asymptotic value of $2 \pi(1-\varpi / \rho)$ (see Fig. 11). Note also the red/blue shift $\exp (\sigma)$ between the core and the outer regions. In the next section, the behaviour of the $4 D$ tensor waves in this background is investigated, both analytically and numerically, and we will find that this system does not support quasi-localized gravity on the brane.

\section{B. Tensor Perturbations}

In this section, we investigate the propagation of tensor perturbations, with respect to the $4 D$ vortex worldvolume, on the background field configuration studied 
in the previous section. For this purpose we derive the equation of motion of tensor perturbations in the Jordan metric, assuming that the scalar and vector degrees of freedom are fixed.

Since we are interested in pure $4 D$ tensor perturbations in the Jordan frame, the perturbed metric (15) reduces to

$$
\mathrm{d} s^{2}=\mathrm{e}^{\sigma}\left(\eta_{\mu \nu}+h_{\mu \nu}\right) \mathrm{d} x^{\mu} \mathrm{d} x^{\nu}+\frac{\mathrm{e}^{\sigma} \mathrm{d} z^{2}+\varpi^{2} \mathrm{~d} \theta^{2}}{m_{\mathrm{h}}^{2}}
$$

where $h_{\mu \nu}$ is a transverse and traceless tensor

$$
\eta^{\mu \beta} \nabla_{\beta} h_{\mu \nu}=0, \quad \eta^{\mu \nu} h_{\mu \nu}=0
$$

and $z$ is a dimensionless conformal coordinate defined by

$$
z \equiv \int_{0}^{\rho} \mathrm{e}^{-\sigma(u) / 2} \mathrm{~d} u
$$

With the hyperstring forming fields being of the scalar kind for the Higgs, and of the vector kind for the gauge boson, the tensor modes decouple from the background fields. The perturbed equations of motion are obtained by perturbing the Einstein-Jordan equations (12) at first order. From the perturbed metric tensor $\delta g_{\mu \nu}$ given in Eq. (35), one obtains in terms of the dimensionless quantities (see Appendix B]

$$
h_{\mu \nu}^{\prime \prime}+\left(\frac{3}{2} \sigma^{\prime}+\frac{\varpi^{\prime}}{\varpi}+\phi^{\prime}\right) h_{\mu \nu}^{\prime}+\tilde{\square} h_{\mu \nu}+\frac{\mathrm{e}^{\sigma}}{\varpi^{2}} \partial_{\theta}^{2} h_{\mu \nu}=0,
$$

where the prime denotes the derivative with respect to $z$ and $\tilde{\square}$ stands for the dimensionless four-dimensional d'Alembertian

$$
\tilde{\square} \equiv \frac{\square}{m_{\mathrm{h}}^{2}}=\frac{\eta^{\mu \nu} \partial_{\mu} \partial_{\nu}}{m_{\mathrm{h}}^{2}}
$$

It is convenient to decompose these perturbations in terms of winding modes around the hyperstring and d'Alembertian eigenfunctions:

$$
h_{\alpha \beta}\left(x^{\mu}, \rho, \theta\right)=\sum_{p} \mathrm{e}^{i p \theta} \int \hat{h}_{\alpha \beta}^{(p)}(\bar{M}, \rho) \Delta_{\bar{M}}\left(x^{\mu}\right) \mathrm{d} \bar{M},
$$

where $\Delta_{\bar{M}}$ verifies

$$
\left(\tilde{\square}+\bar{M}^{2}\right) \Delta_{\bar{M}}=0 .
$$

Moreover, the rescaling

$$
\xi_{\alpha \beta}^{(p)}=\mathrm{e}^{3 \sigma / 4+\phi / 2} \sqrt{\varpi} \hat{h}_{\alpha \beta}^{(p)},
$$

allows to recast Eq. (38) in a Schrödinger-like form for the $\xi_{\alpha \beta}^{(p)}$ modes

$$
-\frac{\mathrm{d}^{2} \xi}{\mathrm{d} z^{2}}+V_{p}(z) \xi=\bar{M}^{2} \xi
$$

where the tensor and angular mode indexes have been removed. The potential $V_{p}$ is given by

$$
V_{p}=W^{2}+W^{\prime}+\frac{\mathrm{e}^{\sigma}}{\varpi^{2}} p^{2}
$$

with

$$
W(z)=\frac{3}{4} \sigma^{\prime}+\frac{1}{2} \frac{\varpi^{\prime}}{\varpi}+\frac{1}{2} \phi^{\prime}
$$

In Eq. (43), $\bar{M}^{2}$ stands for the dimensionless d'Alembertian eigenvalue

$$
\bar{M}^{2} \equiv \frac{M^{2}}{m_{\mathrm{h}}^{2}}=-\frac{\eta^{\alpha \beta} k_{\alpha} k_{\beta}}{m_{\mathrm{h}}^{2}} .
$$

Note that for the zero angular momentum modes $p=0$, Eq. (43) is supersymmetric and $W$ is the superpotential [4, 44]. Indeed, defining the operator

$$
\mathcal{A} \equiv \frac{\mathrm{d}}{\mathrm{d} z}+W(z)
$$

simplifies Eq. (43) into

$$
\left(\mathcal{A} \mathcal{A}^{\dagger}+\frac{\mathrm{e}^{\sigma}}{\varpi^{2}} p^{2}\right) \xi=\bar{M}^{2} \xi
$$

This ensures that all the eigenvalues $\bar{M}^{2}$ are positive, and thus there are no tachyons in this model, provided supersymmetry is not broken, i.e. there exists a normalizable zero mode.

\section{Quasi-Localized States and $4 D$ Gravity}

\section{Pure Cylindrical Waves}

It is interesting to briefly recall the case of cylindrical waves propagating in flat spacetime. In that case, without dilaton, $\sigma=\phi=0$ and $\varpi=z$, the potential (44) simplifies into

$$
V_{p}=-\frac{1}{4 z^{2}}+\frac{p^{2}}{z^{2}},
$$

which is always negative definite for $p=0$ and positive definite otherwise. Eq. (43) is a Bessel equation whose regular solutions in the hyperstring core are

$$
\xi^{(p)}(\bar{M}, z) \propto \sqrt{z} \mathrm{~J}_{p}(\bar{M} z),
$$

the $\mathrm{Y}_{p}$ and $\mathrm{K}_{p}$ modes being singular in $z=0$ whereas the $\mathrm{I}_{p}$ modes are singular at infinity. Moreover, since [45]

$$
\mathrm{J}_{p}(z) \underset{0}{\sim} \frac{1}{\Gamma(p+1)}\left(\frac{z}{2}\right)^{p}
$$

only the zero angular momentum modes $p=0$ do not vanish in the string core. Note that in the special case 
$\bar{M}=0$ the regular solutions simplify to $z^{p}$. Far from the hyperstring core

$$
\xi^{(p)}(\bar{M} z) \underset{\infty}{\propto} \sqrt{\frac{2}{\pi \bar{M}}} \cos \left(\bar{M} z-p \frac{\pi}{2}-\frac{\pi}{4}\right),
$$

and using plane wave normalization at infinity, one gets from Eq. (42) the spectral density

$$
\left|\hat{h}^{(0)}(\bar{M}, 0)\right|^{2}=\frac{\pi}{2} \bar{M}
$$

These tensor modes therefore contribute to a Newton potential on the brane [11, 12]

$$
V_{\mathrm{g}}(x) \propto \int_{0}^{\infty}|\hat{h}(\bar{M}, 0)|^{2} \frac{\mathrm{e}^{-\bar{M} x}}{x} \mathrm{~d} \bar{M} \propto \frac{1}{x^{3}},
$$

i.e. compatible with the standard Newtonian gravity in a six-dimensional flat spacetime.

As discussed in the introduction, if there exist localized or quasi-localized waves in the hyperstring the spectral density will be modified, and hence so will the resulting potential in Eq. (54).

\section{Gravitational Propagation on the Hyperstring Background}

According to the background solution computed in first section, $\sigma^{\prime}=\phi^{\prime}=0$ and $\varpi^{\prime} / \varpi=1 / z$ both in the hyperstring core and at infinity (see Fig. 11). In these two regions the potential $V_{p}$ behaves like the flat spacetime one and only the zero angular momentum modes $p=0$ can contribute significantly to the spectral density. As can be seen in Fig. 2 significant deviations from the flat case appear in the intermediate region where the derivatives of the background fields are non-vanishing.

However, the potential remains negative definite and does not allow for the existence of bound states or quasibound states in the hyperstring core. In that case, we can use the WKB method to approximate the solutions in the entire extra dimension since the turning points where $\bar{M}^{2}=V_{0}$ do not exist (see Fig. 2)

$$
\xi_{\mathrm{WKB}}(\bar{M}, z) \propto \frac{\exp \left(i \int^{z} \sqrt{\bar{M}^{2}-V_{0}(u)} \mathrm{d} u\right)}{\left[\bar{M}^{2}-V_{0}(z)\right]^{1 / 4}} .
$$

After normalization at infinity one gets, from Eq. (42), a spectral density in the hyperstring core similar to the one induced by the pure cylindrical waves

$$
\left|\hat{h}_{\bar{M}}(0)\right|^{2}=\mathrm{e}^{-\phi(0)} \bar{M}
$$

and thus a $6 D$ Newtonian potential, albeit with a weaker gravitational coupling.

We have plotted in Fig. 3 the values of $\hat{h}(\bar{M}, 0)$ obtained from numerical integrations of Eq. (43) for a wide

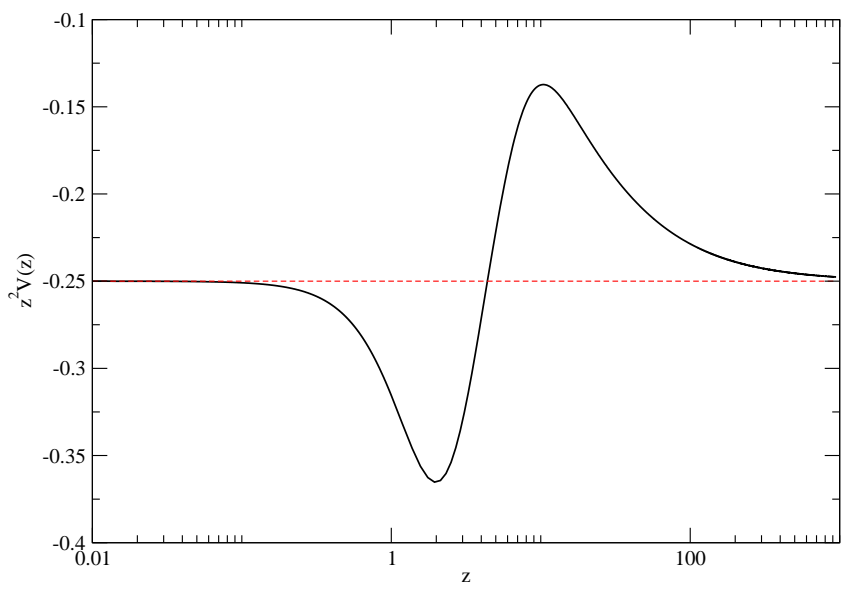

FIG. 2: The potential $z^{2} V_{0}(z)$ governing the behaviour of the tensor perturbations around the hyperstring according to Eq. (43). The background fields are those of Fig 1 and the straight line represents the pure flat spacetime case.

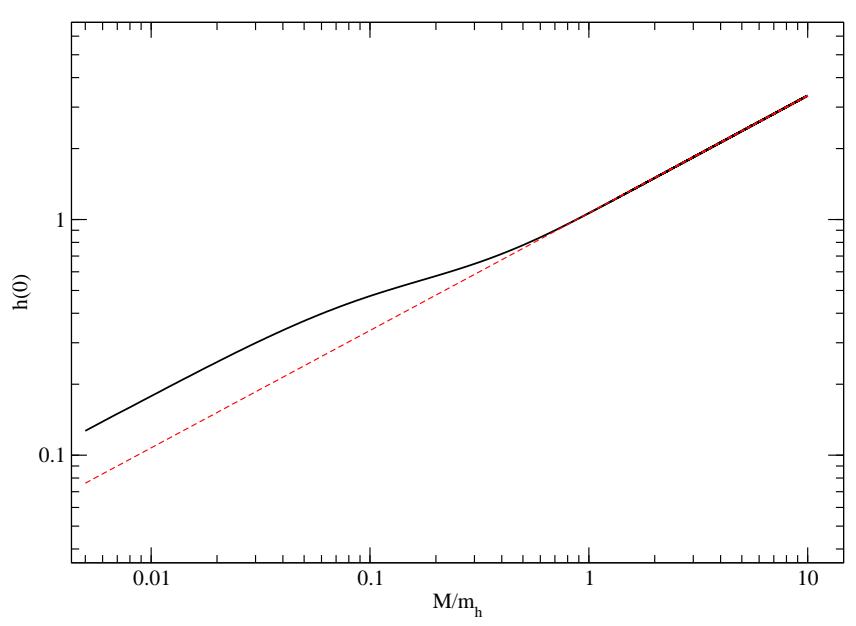

FIG. 3: Normalized values of $\hat{h}(\bar{M}, 0)$ as function of $\bar{M}$ (solid line). For high and small values of $\bar{M}$, one recovers the $\sqrt{\bar{M}}$ behaviour (dashed line). As expected from the WKB approximation, only small deviations from the $6 D$ gravity appear as long as the potential does not allow bound states.

range of masses $\bar{M}$. This confirms the WKB result, with only slight deviations in the intermediate range.

According to the previous discussions, this is physically expected as long as the potential $V_{0}$ remains negative definite: there are no metastable $4 D$ tensor waves inside the core that could induce peaks in the spectral density. In the next section, we search for the precise conditions under which the potential would in fact become positive for a general codimension $N_{\mathrm{c}}=2$ defect and generic dilaton potential. 


\section{Confining Potentials and the Dominant Energy Condition}

According to Eqs. (44) and (45), the potential $V_{0}$ driving the propagation of the non-winding $(p=0) 4 D$ tensor modes in the bulk reads

$$
2 V_{0}=\frac{1}{2}\left(\frac{3}{2} \sigma^{\prime}+\frac{\varpi^{\prime}}{\varpi}+\phi^{\prime}\right)^{2}-\frac{\varpi^{2}}{\varpi^{2}}+\frac{3}{2} \sigma^{\prime \prime}+\frac{\varpi^{\prime \prime}}{\varpi}+\phi^{\prime \prime} .
$$

From the topological defect stress tensor components (19), one can define the dimensionless matter energy $\mathcal{E}$ and pressure $\mathcal{P}$ by

$$
\begin{aligned}
& \mathcal{E} \equiv \frac{1}{2}\left[\dot{f}^{2}+\frac{f^{2} Q^{2}}{\varpi^{2}}+\frac{1}{4}\left(f^{2}-1\right)^{2}+\frac{\dot{Q}^{2}}{\varepsilon \varpi^{2}}\right], \\
& \mathcal{P} \equiv \frac{1}{2}\left[\dot{f}^{2}-\frac{f^{2} Q^{2}}{\varpi^{2}}-\frac{1}{4}\left(f^{2}-1\right)^{2}+\frac{\dot{Q}^{2}}{\varepsilon \varpi^{2}}\right] .
\end{aligned}
$$

The second order derivatives in Eq. (57) can be expressed in terms of first order derivatives by means of the dynamical Einstein-Jordan equation (20). In terms of the conformal coordinate $z$ and $\hat{U} \equiv \exp (\sigma) U / m_{\mathrm{h}}^{2}$, Eq. (20) yields

$$
\begin{aligned}
\frac{3}{2} \sigma^{\prime \prime} & +\frac{\varpi^{\prime \prime}}{\varpi}+\phi^{\prime \prime}=-2 \alpha \mathrm{e}^{\sigma-\phi} \mathcal{E}-\frac{1}{2} \hat{U}-\frac{3}{4} \sigma^{2}-\sigma^{\prime} \frac{\varpi^{\prime}}{\varpi} \\
& -\frac{3}{2} \phi^{\prime 2}-\left(\sigma^{\prime}+\frac{\varpi^{\prime}}{\varpi}\right) \phi^{\prime}
\end{aligned}
$$

Making use of the constraint equation (21)

$$
\begin{aligned}
\frac{3}{2} \sigma^{\prime 2} & -\frac{1}{2} \phi^{\prime 2}+2 \sigma^{\prime} \frac{\varpi^{\prime}}{\varpi}+\left(2 \sigma^{\prime}+\frac{\varpi^{\prime}}{\varpi}\right) \phi^{\prime} \\
& =2 \alpha \mathrm{e}^{\sigma-\phi} \mathcal{P}-\frac{1}{2} \hat{U}
\end{aligned}
$$

the potential $V_{0}$ simplifies to

$$
2 V_{0}=-2 \alpha \mathrm{e}^{\sigma-\phi}(\mathcal{E}-\mathcal{P})-\frac{1}{2}\left(\frac{3}{2} \sigma^{\prime}+\frac{\varpi^{\prime}}{\varpi}+\phi^{\prime}\right)^{2}-\hat{U}
$$

which, according to Eq. (58), is negative for positive dilatonic potentials $U$. In fact it is clear in Eq. (61) that, for a general defect in codimension two, either $\mathcal{E}-\mathcal{P}<0$ or $\hat{U}<0$ are required for $V_{0}$ to become positive at some point. The former condition would violate the Dominant Energy Condition (DEC) for the matter stress tensor, and one would need "special" hyperstring forming fields that support tachyonic propagation [46]. It is beyond the scope of this work to discuss more fundamental motivations and consequences of negative dilatonic potentials [47], however we note that the simplest example of this is achieved by choosing a negative cosmological constant [see Eq. (7)]. In absence of a dilaton, the six-dimensional spacetime generated by a hyperstring in the presence of a negative cosmological constant has in fact already been studied in Refs. [29, 48, 49] and explicitly realizes Randall-Sundrum gravity confinement. However, such defect models require strong fine-tunings on the model parameters, and stay in the realm of finite volume extra-dimensions. It is worth pointing out that in this case, since the potential is asymptotically positive due to the negative cosmological constant, there is a normalizable zero mode (reminiscent of the trapped massless graviton of the Randall-Sundrum model), which ensures that the potential $V_{0}$ does not break supersymmetry. Interestingly, one may also expect to confine a discrete spectrum of massive gravitons.

In the next section, we explore the conditions under which there exist quasi-localized $4 D$ tensor waves on a classical defect in higher codimensions, concentrating on the case in which the extra dimensional space is isotropic.

\section{CLASSICAL DEFECTS IN ISOTROPIC EXTRA DIMENSIONS}

The action (17) can be generalized in a straightforward way to a $N$-dimensional spacetime and the EinsteinJordan equations remain given by Eqs. (12) and (13). The dynamics in the matter sector is driven by the Lagrangian $\mathcal{L}_{\text {mat }}$ which should involve the required fields and their interactions to allow the formation of a $4 D$ topological defect in $N$ dimensions. In the simple case where the defect forming fields do not break the $4 D$ Poincaré invariance, i.e. there is no current flowing along the defect [50, 51, 52, 53], the $4 D$ components of the stress tensor verify

$$
T_{\mu \nu}=-\mathcal{E} g_{\mu \nu}
$$

where $\mathcal{E}$ is a function of the transverse coordinates only. Like the other components of the stress tensor, the profile of $\mathcal{E}$ in the extra dimensions, and consequently the behaviour of the metric fluctuations $\delta g_{\mu \nu}$, depends of the underlying model defined by $\mathcal{L}_{\text {mat }}$.

Here, we consider the generalization of Eq. (15) to more than two isotropic extra dimensions [54, 55]

$$
\mathrm{d} s^{2}=\mathrm{e}^{\sigma(z)} \eta_{\mu \nu} \mathrm{d} x^{\mu} \mathrm{d} x^{\nu}+\mathrm{e}^{\sigma(z)} \mathrm{d} z^{2}+\omega^{2}(z) \mathrm{d} \Omega_{n}^{2},
$$

where

$$
\mathrm{d} \Omega_{n}^{2}=\sum_{i=1}^{n} \Upsilon_{i}\left(\theta_{j<i}\right) \mathrm{d} \theta_{i}^{2}
$$

is the interval of a $n$ dimensional maximally symmetric space. Depending on the curvature $k$ of this subspace, one may choose the coordinates such as $\Upsilon_{1}=1$ and

$$
\Upsilon_{i}\left(\theta_{j<i}\right) \equiv \Upsilon_{2}\left(\theta_{1}\right) \prod_{j=2}^{i-1} \sin ^{2}\left(\theta_{j}\right)
$$

where $\Upsilon_{2}$ is $\sin ^{2}\left(\theta_{2}\right), \theta_{2}^{2}$ or $\sinh ^{2}\left(\theta_{2}\right)$ for positive, null and vanishing curvature $k$, respectively. 
In this case, the Einstein-Jordan equations (12) read

$$
\begin{aligned}
\frac{3}{2} \sigma^{\prime \prime}+n \frac{\omega^{\prime \prime}}{\omega}+\phi^{\prime \prime} & =-\kappa_{N}^{2} \mathrm{e}^{\sigma-\phi} \mathcal{E}-\frac{1}{2} \mathrm{e}^{\sigma} U+\frac{\mathrm{e}^{\sigma}}{\omega^{2}} \frac{n(n-1)}{2} k \\
& -\frac{3}{4} \sigma^{\prime 2}-\frac{n(n-1)}{2} \frac{\omega^{\prime 2}}{\omega^{2}}-\frac{3}{2} \phi^{\prime 2} \\
& -n \sigma^{\prime} \frac{\omega^{\prime}}{\omega}-\left(\sigma^{\prime}+n \frac{\varpi^{\prime}}{\varpi}\right) \phi^{\prime},
\end{aligned}
$$

for the $(\mu \nu)$ components, while the constraint equation $(z z)$ is

$$
\begin{aligned}
\frac{3}{2} \sigma^{\prime 2} & -\frac{1}{2} \phi^{\prime 2}+\frac{n(n-1)}{2} \frac{\omega^{\prime 2}}{\omega^{2}}+2 n \sigma^{\prime} \frac{\omega^{\prime}}{\omega}-\frac{\mathrm{e}^{\sigma}}{\omega^{2}} \frac{n(n-1)}{2} k \\
+ & \left(2 \sigma^{\prime}+n \frac{\varpi^{\prime}}{\varpi}\right) \phi^{\prime}=\kappa_{N}^{2} \mathrm{e}^{\sigma-\phi} \mathcal{P}-\frac{1}{2} \mathrm{e}^{\sigma} U
\end{aligned}
$$

with $\mathcal{P}$ the pressure along the $z$ extra dimension. The tensor perturbations $\delta g_{\mu \nu}=\exp (\sigma) h_{\mu \nu}$ around this metric end up being solutions of

$$
h_{\mu \nu}^{\prime \prime}+\left(\frac{3}{2} \sigma^{\prime}+n \frac{\omega^{\prime}}{\omega}+\phi^{\prime}\right) h_{\mu \nu}^{\prime}+\tilde{\square} h_{\mu \nu}+\frac{\mathrm{e}^{\sigma}}{\omega^{2}} \mathrm{~L}_{n} h_{\mu \nu}=0,
$$

where $\mathrm{L}_{n}$ is a generalized "angular" differential operator, e.g. for $k=1$ one recovers the hyperspherical Laplacian

$$
\mathrm{L}_{n}=\sum_{i=1}^{n} \frac{1}{\Upsilon_{i}}\left(\partial_{\theta_{i}}^{2}+\frac{n-i}{\tan \theta_{i}} \partial_{\theta_{i}}\right)
$$

For the zero angular momentum modes, i.e. those which are non-vanishing on the brane for symmetry reasons, one recovers the Schrödinger equation (43) for the rescaled quantity

$$
\xi_{\alpha \beta}=\mathrm{e}^{3 \sigma / 4+\phi / 2} \omega^{n / 2} \hat{h}_{\alpha \beta} .
$$

Using the above Einstein-Jordan equations, as described in Sect. IID the potential is found to be

$$
\begin{aligned}
2 V_{0} & =-\kappa_{N}^{2} \mathrm{e}^{\sigma-\phi}(\mathcal{E}-\mathcal{P})-\mathrm{e}^{\sigma} U+\frac{\mathrm{e}^{\sigma}}{\omega^{2}} n(n-1) k \\
& -\frac{1}{2}\left(\frac{3}{2} \sigma^{\prime}+n \frac{\omega^{\prime}}{\omega}+\phi^{\prime}\right)^{2} \cdot
\end{aligned}
$$

In contrast to the hyperstring potential studied before [see Eq. (61)], it appears that for positive curvature $k=1$ the potential may now take positive values, whereas for $k=0,-1$, one would still need to violate the DEC to allow for quasi-localized states. This result is not really surprising, as often in General Relativity, a standard way to mimic matter that violates positive energy conditions is to consider positive curvature space (e.g. see Refs. 20, 56, 57, 58]). From Eq. (63), note that $k$ is the curvature of $n=N_{\mathrm{c}}-1$ extra dimensional subspace and, according to the asymptotic behaviour of $\omega(z)$, the volume of the total $N_{\mathrm{c}}$ extra dimensions may be infinite even for $k=1$.
Although we have not presented the existence of solitonic solutions compatible with the metric (63), the condition $k=1$ may be generically fulfilled. For example the case of $N_{\mathrm{c}}=3$ extra dimensions with a positive curvature $k$ may be realized by a hypermonopole [19, 54, 59]. In such a framework one may indeed expect to find quasilocalized tensor modes in the core without violating the DEC 60].

\section{CONCLUSION}

We have discussed in this paper the conditions under which one can realize quasi-localized gravity using underlying topological defect models. For defects with codimension $N_{\mathrm{c}}=2$ the existence of metastable tensor modes requires violation of the Dominant Energy Condition by the defect matter. For codimension $N_{\mathrm{c}}>2$ we found that this requirement may be relaxed for appropriate defect matter. In this case the background solution can, at least in principle, support metastable gravity. The explicit construction of $N_{\mathrm{c}}>2$ codimensional sigma models that quasi-localize gravity is subject to future work [60].

\section{Acknowledgments}

We thank Gregory Gabadadze, Patrick Peter, Massimo Porrati and Jean-Philippe Uzan for enlightening discussions and useful comments. J-W. R. thanks the I.A.P., where part of this work was done, for their warm hospitality.

\section{APPENDIX A: RELAXATION METHOD}

To solve the set of differential equations (20), (22), (23), (29) and (30), we have used a relaxation method 61]. In terms of dimensionless fields and parameters, the action (17) can be recast into

$$
\begin{aligned}
-\frac{2 \alpha}{\pi \eta^{2}} S & =\int \mathrm{e}^{\phi}\left[-3 \dot{\gamma}^{2} \varpi-4 \gamma \dot{\gamma} \dot{\varpi}+\gamma^{2} \varpi\left(\dot{\phi}^{2}+\beta \phi^{2}\right)\right. \\
& \left.-\dot{\phi}\left(4 \gamma \dot{\gamma} \varpi+2 \dot{\varpi} \gamma^{2}\right)\right] \mathrm{d} \rho+2 \alpha \int \gamma^{2} \varpi\left[\dot{f}^{2}\right. \\
& \left.+\frac{f^{2} Q^{2}}{\varpi^{2}}+\frac{1}{4}\left(f^{2}-1\right)^{2}+\frac{\dot{Q}^{2}}{\varepsilon \varpi^{2}}\right] \mathrm{d} \rho,
\end{aligned}
$$

with $\gamma=\mathrm{e}^{\sigma}$, and where integrations by parts have been used to keep only first order derivatives in the metric factors. After discretization of the radial coordinates $\rho$, the discrete action reads $\tilde{S}=\tilde{S}_{\mathrm{g}}+\tilde{S}_{\mathrm{m}}$ with 


$$
\begin{aligned}
-\frac{2 \alpha}{\pi \eta^{2}} \tilde{S}_{\mathrm{g}} & =\sum_{i} \mathrm{e}^{\phi_{i}}\left[-3 \varpi_{i} \frac{\left(\gamma_{i+1 / 2}-\gamma_{i-1 / 2}\right)^{2}}{h}-4 \gamma_{i} \frac{\left(\gamma_{i+1 / 2}-\gamma_{i-1 / 2}\right)\left(\varpi_{i+1 / 2}-\varpi_{i-1 / 2}\right)}{h}+\gamma_{i}^{2} \varpi_{i} \frac{\left(\phi_{i+1 / 2}-\phi_{i-1 / 2}\right)^{2}}{h}\right. \\
& \left.-4 \gamma_{i} \varpi_{i} \frac{\left(\gamma_{i+1 / 2}-\gamma_{i-1 / 2}\right)\left(\phi_{i+1 / 2}-\phi_{i-1 / 2}\right)}{h}-2 \gamma_{i}^{2} \frac{\left(\varpi_{i+1 / 2}-\varpi_{i-1 / 2}\right)\left(\phi_{i+1 / 2}-\phi_{i-1 / 2}\right)}{h}+h \beta \gamma_{i}^{2} \varpi_{i} \phi_{i}^{2}\right],
\end{aligned}
$$

in the gravity sector, and

$$
-\frac{\tilde{S}_{\mathrm{m}}}{\pi \eta^{2}}=\sum_{i}\left[\gamma_{i}^{2} \varpi_{i} \frac{\left(f_{i+1 / 2}-f_{i-1 / 2}\right)^{2}}{h}+h \gamma_{i}^{2} \frac{f_{i}^{2} Q_{i}^{2}}{\varpi_{i}}+h \frac{\gamma_{i}^{2} \varpi_{i}}{4}\left(f_{i}^{2}-1\right)^{2}+\frac{\gamma_{i}^{2}}{\varepsilon \varpi_{i}} \frac{\left(Q_{i+1 / 2}-Q_{i-1 / 2}\right)^{2}}{h}\right],
$$

for the matter sector. The grid resolution is given by $h=$ $\rho_{i+1}-\rho_{i}$ where the index $i$ indicates that the fields have to be evaluated at the discrete points $\rho_{i}$. The discrete derivatives have been expressed in their centered form, e.g.

$$
\dot{f}_{i}=\frac{f_{i+1 / 2}-f_{i-1 / 2}}{h}+\mathcal{O}\left(h^{3}\right)
$$

where $f_{i+1 / 2}$ is evaluated on a half step shifted mesh. By differentiating the discrete action $\tilde{S}$ with respect to the discrete fields $\gamma_{i}, \varpi_{i}, \phi_{i}, f_{i}$ and $Q_{i}$, one gets the finite difference equations corresponding to Eqs. (20), (22), (23), (29) and (30), respectively. From an initial guess of all the discrete fields $\overrightarrow{\mathcal{F}}_{i}^{0}$ on the grid $\rho_{i}$, the solutions of the finite difference equations are obtained by a successive over-relaxation method. Here $\overrightarrow{\mathcal{F}}_{i}$ designs a five dimensional vector whose components are the discrete fields $\gamma_{i}, \varpi_{i}, \phi_{i}, f_{i}$ and $Q_{i}$. At step $p+1$, the fields are updated by a Newton's method to reduce the error with respect to the true solution:

$$
\overrightarrow{\mathcal{F}}_{i}^{p+1}=\overrightarrow{\mathcal{F}}_{i}^{p}+s \delta \overrightarrow{\mathcal{F}}_{i}^{p},
$$

where $\delta \overrightarrow{\mathcal{F}}_{i}^{p}$ is computed to solve

$$
\overrightarrow{\mathcal{E}}\left(\overrightarrow{\mathcal{F}}_{i}^{p+1}\right) \simeq \overrightarrow{\mathcal{E}}\left(\overrightarrow{\mathcal{F}}_{i}^{p}\right)+\vec{\nabla} \overrightarrow{\mathcal{E}}\left(\delta \overrightarrow{\mathcal{F}}_{i}^{p}\right)=0
$$

$\overrightarrow{\mathcal{E}}$ being the finite difference equations. In Eq. (A5), $s$ is the over-relaxation factor. The boundary conditions are part of the finite difference equations $\overrightarrow{\mathcal{E}}$ since they appear as the constraints which have to be satisfied by $\overrightarrow{\mathcal{F}}_{1}^{p}$ and $\overrightarrow{\mathcal{F}}_{N}^{p}$, where $N$ is the total number of points of the $\rho_{i}$ mesh. The iterative process is stopped when the discretized action $\tilde{S}$ given by Eqs. (A2) and (A3) remains stationary at the machine precision. Moreover, we have also verified that the relaxed solutions satisfy the constraint equation (21). The relaxed fields have been plotted in Fig. 1 for an assumed generic set of parameters $\alpha, \beta$ and $\epsilon$.

\section{APPENDIX B: PERTURBED QUANTITIES}

For the metric (63), the only non-vanishing tensor perturbations are

$$
\delta g_{\mu \nu}=\mathrm{e}^{\sigma} h_{\mu \nu}, \quad \delta g^{\mu \nu}=-\mathrm{e}^{-\sigma} \eta^{\mu \alpha} \eta^{\nu \beta} h_{\alpha \beta},
$$

from which the perturbed Christoffel symbols read

$$
\begin{aligned}
\delta \Gamma_{\mu \nu}^{\alpha} & =\frac{1}{2} \eta^{\alpha \beta}\left(h_{\beta \mu, \nu}+h_{\beta \nu, \mu}-h_{\mu \nu, \beta}\right), \\
\delta \Gamma_{\mu z}^{\alpha} & =\frac{1}{2} \eta^{\alpha \beta} h_{\mu \beta}^{\prime}, \\
\delta \Gamma_{\mu \theta_{i}}^{\alpha} & =\frac{1}{2} \eta^{\alpha \beta} \partial_{\theta_{i}} h_{\mu \beta}, \\
\delta \Gamma_{\mu \nu}^{z} & =-\frac{1}{2}\left(h_{\mu \nu}^{\prime}+\sigma^{\prime} h_{\mu \nu}\right), \\
\delta \Gamma_{\mu \nu}^{\theta_{i}} & =-\frac{1}{2} \frac{\mathrm{e}^{\sigma}}{\omega^{2}} \frac{\partial_{\theta_{i}} h_{\mu \nu}}{\Upsilon_{i}} .
\end{aligned}
$$

From the perturbed Ricci tensor

$$
\begin{aligned}
\delta R_{B D} & =-\delta \Gamma_{B A, D}^{A}+\delta \Gamma_{B D, A}^{A}+\Gamma_{B D}^{H} \delta \Gamma_{A H}^{A}-\Gamma_{D H}^{A} \delta \Gamma_{B A}^{H} \\
& -\Gamma_{B A}^{H} \delta \Gamma_{D H}^{A}+\Gamma_{A H}^{A} \delta \Gamma_{B D}^{H},
\end{aligned}
$$

the non-vanishing components of the perturbed Einstein tensor read

$$
\begin{aligned}
\delta G_{\mu \nu} & =-\frac{1}{2}\left\{h_{\mu \nu}^{\prime \prime}+\left(\frac{3}{2} \sigma^{\prime}+n \frac{\omega^{\prime}}{\omega}\right) h_{\mu \nu}^{\prime}+\square h_{\mu \nu}\right. \\
& +\frac{\mathrm{e}^{\sigma}}{\omega^{2}} \mathrm{~L}_{n} h_{\mu \nu}-\left[3 \sigma^{\prime \prime}+2 n \frac{\omega^{\prime \prime}}{\omega}+\frac{3}{2} \sigma^{\prime 2}\right. \\
& \left.\left.+n(n-1) \frac{\omega^{\prime 2}}{\omega^{2}}+2 n \sigma^{\prime} \frac{\omega^{\prime}}{\omega}-\frac{\mathrm{e}^{\sigma}}{\omega^{2}} n(n-1) k\right] h_{\mu \nu}\right\} .
\end{aligned}
$$

From Eqs. (12) and (B1), the perturbed dilatonic source terms, i.e. other than the matter stress tensor, are

$$
\begin{aligned}
\delta \mathcal{D}_{\mu \nu} & =\frac{1}{2} \phi^{\prime} h_{\mu \nu}^{\prime}-\left[\phi^{\prime \prime}+\frac{3}{2} \phi^{2}+\left(\sigma^{\prime}+n \frac{\omega^{\prime}}{\omega}\right) \phi^{\prime}\right. \\
& \left.+\frac{1}{2} U(\phi)\right] h_{\mu \nu},
\end{aligned}
$$


whereas $\delta T_{\mu \nu}$ is readily obtained from Eq. (62), or Eq. (19) for the Abelian Higgs vortex. From Eqs. (B4), and (B5), one obtains Eqs. (38) and (68), up to the background Einstein-Jordan equation (12).
[1] G. R. Dvali, G. Gabadadze, and M. Porrati, Phys. Lett. B485, 208 (2000), hep-th/0005016.

[2] I. Antoniadis, R. Minasian, and P. Vanhove, Nucl. Phys. B648, 69 (2003), hep-th/0209030.

[3] E. Kohlprath, Nucl. Phys. B697, 243 (2004), hepth/0311251.

[4] E. Kohlprath and P. Vanhove (2004), hep-th/0409197.

[5] C. Deffayet, Phys. Lett. B502, 199 (2001), hepth/0010186.

[6] G. R. Dvali and G. Gabadadze, Phys. Rev. D63, 065007 (2001), hep-th/0008054.

[7] G. Dvali, G. Gabadadze, X.-r. Hou, and E. Sefusatti, Phys. Rev. D67, 044019 (2003), hep-th/0111266.

[8] G. Dvali, G. Gabadadze, and M. Shifman, Phys. Rev. D67, 044020 (2003), hep-th/0202174.

[9] G. Gabadadze (2004), hep-th/0408118.

[10] G. Gabadadze and M. Shifman, Phys. Rev. D69, 124032 (2004), hep-th/0312289.

[11] M. Kolanovic, M. Porrati, and J.-W. Rombouts, Phys. Rev. D68, 064018 (2003), hep-th/0304148.

[12] M. Kolanovic, Phys. Rev. D67, 106002 (2003), hepth/0301116.

[13] M. Porrati and J. W. Rombouts (2004), hep-th/0401211.

[14] L. Randall and R. Sundrum, Phys. Rev. Lett. 83, 3370 (1999), hep-ph/9905221.

[15] L. Randall and R. Sundrum, Phys. Rev. Lett. 83, 4690 (1999), hep-th/9906064.

[16] J. Garriga and T. Tanaka, Phys. Rev. Lett. 84, 2778 (2000), hep-th/9911055.

[17] C. Csaki, J. Erlich, and T. J. Hollowood, Phys. Rev. Lett. 84, 5932 (2000), hep-th/0002161.

[18] M. Shaposhnikov, P. Tinyakov, and K. Zuleta (2004), hep-th/0411031.

[19] G. R. Dvali (2000), hep-th/0004057.

[20] S. M. Carroll, J. Geddes, M. B. Hoffman, and R. M. Wald, Phys. Rev. D66, 024036 (2002), hep-th/0110149.

[21] K. Akama, Lect. Notes Phys. 176, 267 (1982), hepth/0001113.

[22] V. A. Rubakov and M. E. Shaposhnikov, Phys. Lett. B125, 136 (1983).

[23] M. Visser, Phys. Lett. B125, 22 (1985).

[24] M. Cvetic and H. H. Soleng, Phys. Rept. 282, 159 (1997), hep-th/9604090.

[25] B. Carter, R. A. Battye, and J.-P. Uzan, Commun. Math. Phys. 235, 289 (2003), hep-th/0204042.

[26] H. Lee and W. S. l'Yi (2000), hep-th/0011144.

[27] F. Bonjour, C. Charmousis, and R. Gregory, Class. Quant. Grav. 16, 2427 (1999), gr-qc/9902081.

[28] C. Ringeval, P. Peter, and J.-P. Uzan, Phys. Rev. D65, 044016 (2002), hep-th/0109194.

[29] P. Peter, C. Ringeval, and J.-P. Uzan (2003), hepth/0301172.

[30] C. Brans and R. H. Dicke, Phys. Rev. 124, 925 (1961).

[31] T. Damour and G. Esposito-Farese, Class. Quant. Grav. 9, 2093 (1992).
[32] A. Riazuelo and J.-P. Uzan, Phys. Rev. D66, 023525 (2002), astro-ph/0107386.

[33] G. F. R. Ellis and J.-P. Uzan (2003), gr-qc/0305099.

[34] M. A. Luty, M. Porrati, and R. Rattazzi, JHEP 09, 029 (2003), hep-th/0303116.

[35] G. Dvali (2004), hep-th/0402130.

[36] A. Nicolis and R. Rattazzi, JHEP 06, 059 (2004), hepth/0404159.

[37] G. Gabadadze and A. Iglesias (2004), hep-th/0407049.

[38] N. K. Nielsen and P. Olesen, Nucl. Phys. B291, 829 (1987).

[39] R. Gregory and C. Santos, Phys. Rev. D56, 1194 (1997), gr-qc/9701014.

[40] Y. Verbin, Phys. Rev. D59, 105015 (1999), hepth/9809002.

[41] Y. Verbin, S. Madsen, A. L. Larsen, and M. Christensen, Phys. Rev. D65, 063503 (2002), gr-qc/0110090.

[42] V. C. de Andrade, P. Peter, and M. E. X. Guimaraes, Phys. Rev. D67, 123509 (2003), gr-qc/0101039.

[43] F. Cooper, A. Khare, and U. Sukhatme, Phys. Rept. 251, 267 (1995), hep-th/9405029.

[44] M. Giovannini, Phys. Rev. D64, 064023 (2001), hepth/0106041.

[45] M. Abramowitz and I. A. Stegun, Handbook of mathematical functions with formulas, graphs, and mathematical tables (National Bureau of Standards, Washington, US, 1970), ninth ed.

[46] R. M. Wald, General Relativity (Univ. Pr., Chicago, 1984).

[47] A. Linde, JHEP 11, 052 (2001), hep-th/0110195.

[48] P. Tinyakov and K. Zuleta, Phys. Rev. D64, 025022 (2001), hep-th/0103062.

[49] M. Giovannini, H. Meyer, and M. E. Shaposhnikov, Nucl. Phys. B619, 615 (2001), hep-th/0104118.

[50] E. Witten, Nucl. Phys. B249, 557 (1985).

[51] B. Carter, Phys. Lett. B228, 466 (1989).

[52] P. Peter, Phys. Rev. D45, 1091 (1992).

[53] B. Carter and P. Peter, Phys. Rev. D52, 1744 (1995), hep-ph/9411425.

[54] C. Csaki, J. Erlich, T. J. Hollowood, and Y. Shirman, Nucl. Phys. B581, 309 (2000), hep-th/0001033.

[55] T. Gherghetta, E. Roessl, and M. E. Shaposhnikov, Phys. Lett. B491, 353 (2000), hep-th/0006251.

[56] J. Martin and P. Peter, Phys. Rev. D68, 103517 (2003), hep-th/0307077.

[57] P. Peter and N. Pinto-Neto, Phys. Rev. D65, 023513 (2002), gr-qc/0109038.

[58] N. Kaloper, J. March-Russell, G. D. Starkman, and M. Trodden, Phys. Rev. Lett. 85, 928 (2000), hep$\mathrm{ph} / 0002001$.

[59] E. Roessl and M. Shaposhnikov, Phys. Rev. D66, 084008 (2002), hep-th/0205320.

[60] C. Ringeval and J.-W. Rombouts, In preparation.

[61] S. L. Adler and T. Piran, Rev. Mod. Phys. 56, 1 (1984). 